\title{
Retrospective review of critical incidents in the post-anaesthesia care unit at a major tertiary hospital
}

\author{
Suze Dominique $\underline{B r u i n s}^{1}$, MBBS, DTM\&H, Pauline Meng Choo Leong ${ }^{2}$, MN, BHSN, Shin Yi $\underline{\text { Ng}^{1}}$, MBBS, MMed
}

\begin{abstract}
INTRODUCTION We reviewed patients with critical incidents that occurred in the post-anaesthesia care unit (PACU) at a major tertiary hospital, and assessed the effect of these incidents on PACU length of stay and discharge disposition. METHODS A retrospective review was conducted of patients in the PACU over a two-year period from 24 June 2011 to 23 August 2013. Data on critical incidents was recorded in the administrative database using a standardised data form. RESULTS There were 701 incidents involving 364 patients; 203 (55.8\%) patients had American Society of Anesthesiologists (ASA) physical status I or II. The most common critical incidents were cardiovascular-related $(n=293,41.8 \%)$, respiratory $(n=155,22.1 \%)$, neurological $(n=52,7.4 \%)$, surgical $(n=47,6.7 \%)$ and airway-related $(n=34,4.9 \%)$. There were two incidents of cardiac arrest and 25 incidents of unexpected reintubations. Many patients $(n=186,51.2 \%)$ stayed for over four hours in the PACU due to critical incidents and $184(50.5 \%)$ patients required a higher level of care postoperatively than initially planned. Some patients $(n=34,9.3 \%)$ returned to the operation theatre for further management. A proportion of patients $(n=64,17.6 \%$ ) had unplanned intensive care unit admissions due to adverse events in the PACU.

CONCLUSION A wide spectrum of critical incidents occur in the PACU, many of which are related to the cardiovascular and respiratory systems. Critical incidents have a major impact on healthcare utilisation and result in prolonged PACU stays and higher levels of postoperative care than initially anticipated.
\end{abstract}

Keywords: critical incidents, length of stay, post-anaesthesia care unit, postoperative care, recovery room

\section{INTRODUCTION}

Monitoring of patients in the post-anaesthesia care unit (PACU) has become the accepted level of care in the immediate postoperative period. ${ }^{(1,2)}$ Patients are admitted to the PACU following surgery and closely cared for by the nursing and anaesthetic staff as they recover from the effects of anaesthesia.

Data on critical incidents in the PACU is an invaluable source of information that can be used to anticipate and prevent such incidents, and improve processes of care. The incidence of complications in the PACU has been reported to range from $22.1 \%$ to $30 \%{ }^{(3-5)}$ with minor complications $(22.1 \%)$ occurring more often than major ones $(0.2 \%) .{ }^{(5)}$ Critical incidents occurring in the PACU are often related to the cardiovascular and respiratory systems. ${ }^{(6,7)}$ Although several studies have been published on complications or critical incidents in the PACU, there is a dearth of both local and Asia-specific data.

The objective of this study was to undertake a retrospective review of the PACU critical incident database at Singapore General Hospital (SGH), Singapore, to categorise critical incidents that occurred in the PACU and assess the effect of these incidents on the length of stay and discharge disposition of patients.

\section{METHODS}

Following approval from the institutional review board, we reviewed patient data that had been documented in SGH's PACU critical incidents database between 24 June 2011 and 23 August 2013. SGH is a 1,597-bed academic tertiary teaching hospital.

A PACU critical incident report was made by the nursing staff if the events met any of the following inclusion criteria: cardiac arrest, intubation/ventilation, inotropic support, allergic reactions, PACU length of stay $>4$ hours and postoperative complications resulting in a higher level of care (i.e. high dependency area/intensive care unit). Patients who were transferred directly from the operation theatre (OT) to an intensive care facility and those who underwent procedures in outlying theatre complexes with a separate PACU were excluded from the database. Reports of patients who had a PACU length of stay of over four hours for purely administrative reasons, such as awaiting discharge from an occupied bed elsewhere, were also excluded. The critical incidents were reviewed and documented in a standardised data collection form according to the predefined categories presented in Box 1 . Some patients may have had more than one incident during their stay in the PACU.

Data routinely captured in the critical incident database included patient demographics (e.g. age and gender) and surgical data (e.g. nature of procedure, surgical specialty and length of surgery). PACU-specific data included length of stay, description of critical incident(s) and management, and discharge disposition of the patient. Additional information was captured retrospectively from the electronic pre-anaesthetic charts, including data on ethnicity, weight, body mass index, American Society of Anesthesiologists (ASA) physical status and type of anaesthetic used (i.e. general, regional or local).

Statistical analysis was performed using SPSS Statistics version 17.0 (SPSS Inc, Chicago, IL, USA). All data was reported as mean \pm standard deviation or median (interquartile 


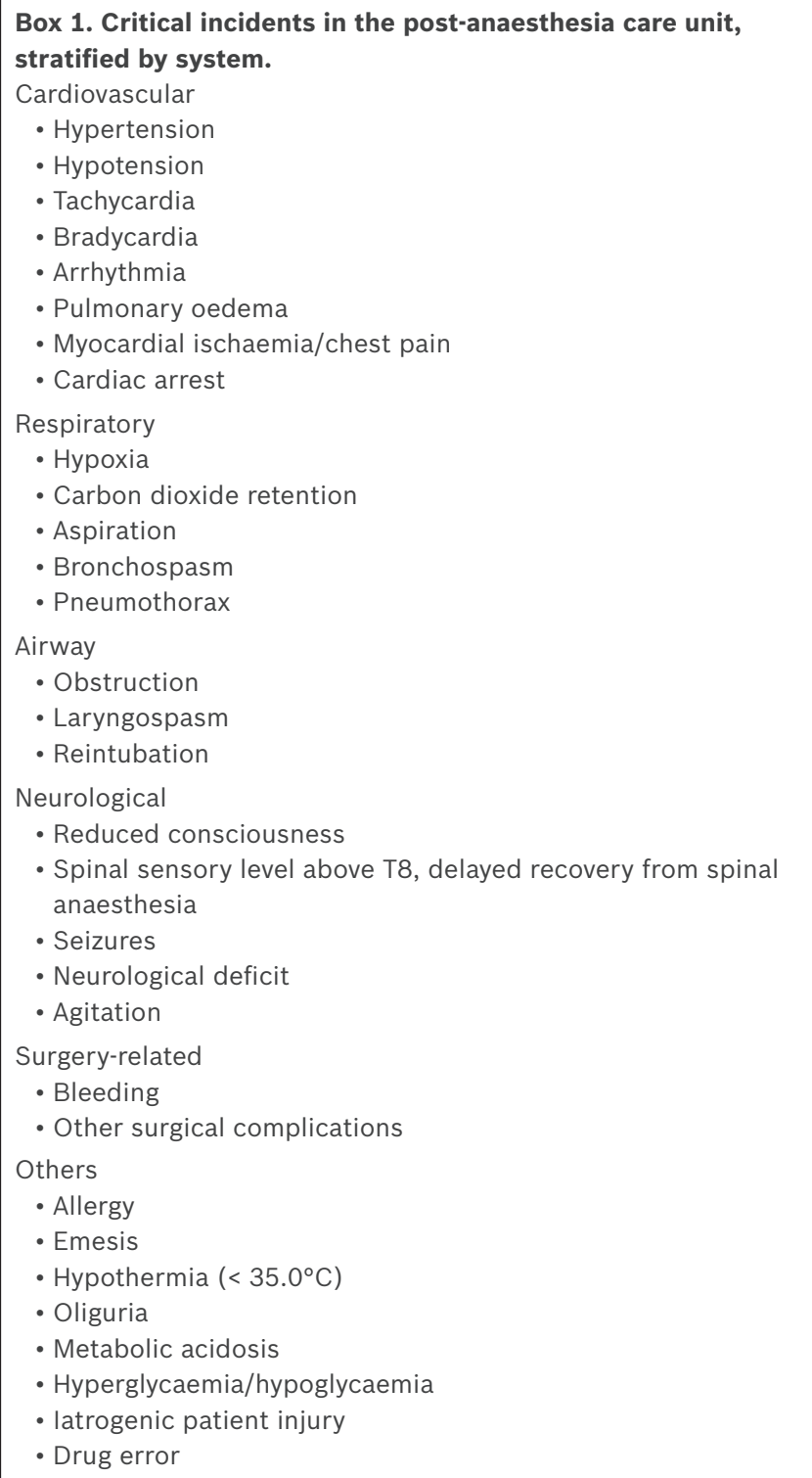

range). Chi-square test was used to analyse categorical data. A p-value $<0.05$ was considered statistically significant.

\section{RESULTS}

During the study period, 49,532 patients underwent procedures in the major OT. Although critical incidents were recorded for 613 patients in the PACU database, 249 patients were excluded from analysis, as their long stay in the PACU was purely for administrative reasons. The remaining 364 patients were analysed. Table I shows the demographic details of patients who underwent procedures in the major OT and those among them with critical incidents in the PACU. The mean age of the patients with critical incidents in the PACU was $62 \pm 16$ (range 16-96) years, and the majority of those patients were of ASA physical status II ( $n=170,46.7 \%)$. The majority of patients with a critical incident were scheduled for elective procedures $(n=271,74.5 \%)$, with the remainder undergoing emergency procedures $(n=93$, $25.5 \%$ ). Patients undergoing emergency procedures were not more likely to have a critical incident when compared to those scheduled for elective procedures (Table II).
Table I. Patient demographics.

\begin{tabular}{|c|c|c|}
\hline \multirow[t]{2}{*}{ Characteristic } & \multicolumn{2}{|c|}{ No. (\%) } \\
\hline & $\begin{array}{c}\text { PACU critical } \\
\text { incident }(n=364)\end{array}$ & Total $(n=49,532)$ \\
\hline \multicolumn{3}{|l|}{ Gender } \\
\hline Female & $202(55.5)$ & $26,439(53.4)$ \\
\hline Male & $162(44.5)$ & $23,093(46.6)$ \\
\hline \multicolumn{3}{|l|}{ Age (yr) } \\
\hline$<18$ & $2(0.5)$ & 665 (1.3) \\
\hline $18-64$ & $190(52.2)$ & $34,874(70.4)$ \\
\hline $65-80$ & $127(34.9)$ & $12,225(24.7)$ \\
\hline$>80$ & $45(12.4)$ & $1,768(3.6)$ \\
\hline \multicolumn{3}{|c|}{ ASA physical status } \\
\hline I and II & $203(55.8)$ & $39,462(79.7)$ \\
\hline III and IV & $161(44.2)$ & 9,854 (19.9) \\
\hline $\mathrm{V}$ and $\mathrm{VI}$ & 0 & $216(0.4)$ \\
\hline
\end{tabular}

Table II. Patients in the PACU stratified by procedure type.

\begin{tabular}{lcccc}
\hline Procedure & \multicolumn{2}{c}{ No. (\%) } & OR* $(95 \% \mathrm{CI})$ & p-value \\
\cline { 2 - 3 } & $\begin{array}{c}\text { Critical } \\
\text { incident } \\
(\mathbf{n}=\mathbf{3 6 4})\end{array}$ & $\begin{array}{c}\text { Total } \\
(\mathbf{n}=\mathbf{4 9 , 5 3 2 )}\end{array}$ & & \\
& $271(74.5)$ & $35,168(71.0)$ & $1.2(0.9-1.5)$ & NS \\
Elective & $93(25.5)$ & $14,364(29.0)$ & $0.8(0.7-1.1)$ & NS \\
\hline
\end{tabular}

*In comparison with emergency procedure. $\mathrm{Cl}$ : confidence interval; NS: not significant; OR: odds ratio; PACU: post-anaesthesia care unit

Most critical incidents occurred in patients who underwent general surgical or orthopaedic procedures $(n=260,71.4 \%$; Table III). Of all procedures that took place in the major OT during the study period, $32.7 \%$ were for general surgical patients and $29.0 \%$ were for orthopaedic patients. Patients from the general surgical and vascular surgical disciplines were associated with a higher PACU critical incident rate when compared to those from other surgical disciplines $(p<0.001)$. Patients undergoing orthopaedic surgery and obstetrics/gynaecology procedures were associated with a lower incidence of PACU critical incidents $(\mathrm{p}<0.001)$.

Among the 364 patients with critical incidents in the PACU, procedures were performed for $322(88.5 \%)$ of them under general anaesthesia; for the remaining patients, regional anaesthesia, local anaesthesia or sedation was used during the procedures. Cardiovascular and respiratory incidents were the most common, with 448 ( $n=701,63.9 \%$ ) incidents being recorded (Fig. 1). Table IV presents a summary of the critical incidents that occurred in the PACU. There were 293 cardiovascular-related critical incidents. The most common cardiovascular incidents were tachycardia $(\mathrm{n}=113,38.6 \%)$, hypotension $(\mathrm{n}=85,29.0 \%)$ and arrhythmia $(\mathrm{n}=31,10.6 \%)$. There were two incidents of cardiac arrest in the PACU, both resulting in death (Table V). Both patients were of ASA physical status III and had undergone emergency procedures.

There were 155 respiratory-related critical incidents in the PACU. Hypoxia $(\mathrm{n}=103,66.5 \%)$ and carbon dioxide $\left(\mathrm{CO}_{2}\right)$ retention ( $n=41,26.5 \%$ ) were the most common respiratory 


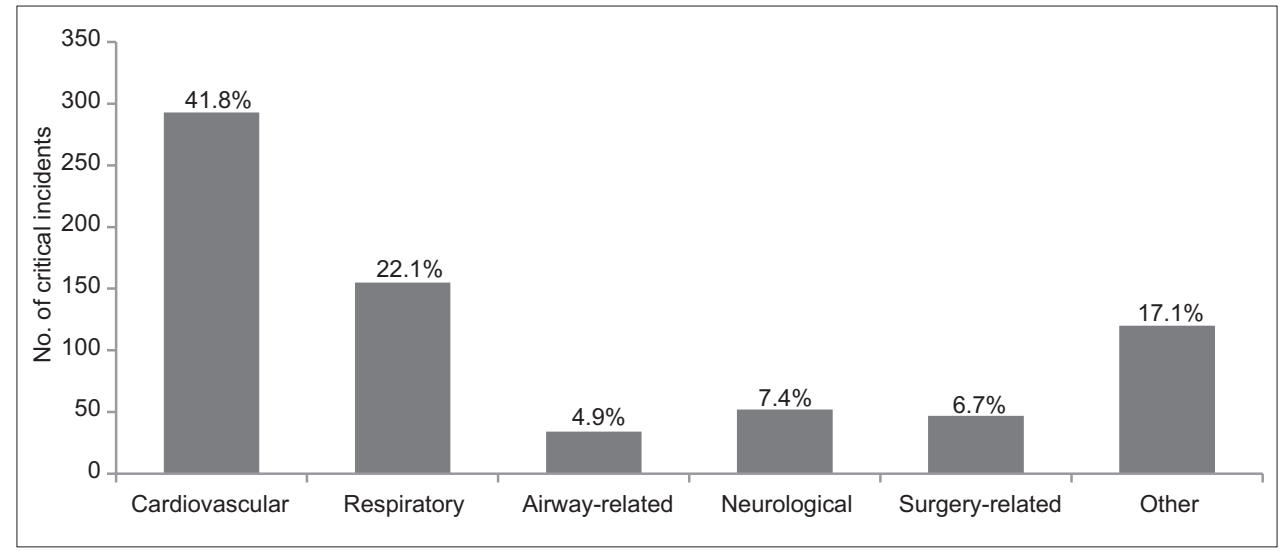

Fig. 1 Bar chart shows classification of post-anaesthesia care unit critical incidents stratified by system $(n=701)$.

Table III. Patients with critical incidents in the PACU stratified by discipline.

\begin{tabular}{|c|c|c|c|c|}
\hline \multirow[t]{2}{*}{ Discipline } & \multicolumn{2}{|c|}{ No. (\%) } & \multirow[t]{2}{*}{$\mathrm{OR}^{*}(95 \% \mathrm{CI})$} & \multirow[t]{2}{*}{ p-value } \\
\hline & Critical incident $(n=364)$ & Total $(n=49,532)$ & & \\
\hline General surgery & $184(50.5)$ & $16,178(32.7)$ & $2.1(1.7-2.6)$ & $<0.001$ \\
\hline Orthopaedic surgery & $76(20.9)$ & $14,350(29.0)$ & $0.6(0.5-0.8)$ & $<0.001$ \\
\hline Plastic surgery & $29(8.0)$ & $4,669(9.4)$ & $0.8(0.6-1.2)$ & NS \\
\hline Cardiothoracic surgery & $19(5.2)$ & $3,272(6.6)$ & $0.8(0.5-1.2)$ & NS \\
\hline Vascular surgery & $16(4.4)$ & $202(0.4)$ & $12.1(7.2-20.4)$ & $<0.001$ \\
\hline Obstetrics and gynaecology & $13(3.6)$ & $5,470(11.0)$ & $0.3(0.2-0.5)$ & $<0.001$ \\
\hline Neurosurgery & $4(1.1)$ & $1,385(2.8)$ & $0.4(0.1-1.0)$ & NS \\
\hline Dental and maxillofacial surgery & $3(0.8)$ & $487(1.0)$ & $0.8(0.3-2.6)$ & NS \\
\hline Gastroenterology & $1(0.3)$ & $33(0.1)$ & $4.2(0.6-31.0)$ & NS \\
\hline Anaesthesia & $1(0.3)$ & $233(0.5)$ & $0.6(0.1-4.2)$ & NS \\
\hline Others & 0 & $307(0.6)$ & - & - \\
\hline
\end{tabular}

${ }^{*}$ Compared to other surgical disciplines. Cl: confidence interval; ENT: ear, nose and throat; NS: not significant; OR: odds ratio; PACU: post-anaesthesia care unit

incidents. There were 25 reintubations, required in 11 patients for Type 1 respiratory failure, ten for Type 2 respiratory failure, two due to cardiac arrest, one for obstruction and one for reduced consciousness. Among this group, 21 patients became unplanned admissions to the intensive care unit (ICU), one patient went to the high dependency area (HD) and one returned to the ward after making a full recovery in the PACU. Two patients died following cardiac arrest. For critical incidents of neurological origin $(n=52)$, the most common incident was reduced consciousness $(n=34$, $65.4 \%$ ) followed by agitation ( $n=9,17.3 \%$ ).

The most frequent surgery-related critical incident in the PACU $(\mathrm{n}=47)$ was bleeding $(\mathrm{n}=37,78.7 \%)$ and 34 patients returned to the OT for further management of their surgical complication. Among the 120 other critical incidents, there were 37 critical incidents of hypothermia (30.8\%) and 28 of metabolic acidosis $(23.3 \%)$. Other notable incidents included allergic reactions $(n=14,11.7 \%)$, iatrogenic patient injury $(n=9,7.5 \%)$ and drug error ( $n=5,4.2 \%)$.

Among the 363 patients who had available data, the majority ( $n=186,51.2 \%$ ) stayed for over four hours in the PACU as a result of these critical incidents. The mean length of stay for the entire cohort was $255 \pm 157$ minutes (4 hours 15 minutes \pm 2 hours 37 minutes). Many patients ( $n=184,50.5 \%$ ) also required a higher level of care than initially planned (Table VI). There were 64 unplanned ICU admissions as a result of critical incidents in the PACU.

\section{DISCUSSION}

There were 701 critical incidents in 364 patients over a 26-month period at our institution. Most critical incidents occurred in ASA physical status II patients, who also made up the largest proportion of patients in this study. The majority of patients were elective patients and underwent general surgical or orthopaedic procedures. Cardiorespiratory incidents comprised the majority of incidents reported, with tachycardia and hypoxia being the most common. Critical incidents resulted in prolonged stays in the PACU and unexpected admissions to higher levels of care, such as the HD and ICU.

Most patients (55.8\%) with critical incidents were of ASA physical status I or II, suggesting that significant critical incidents may occur in patients with relatively few comorbidities and in whom clinicians might not expect the occurrence of critical incidents. A prospective study by Hines et al involving more than 18,000 patients, which aimed to identify adverse events occurring in the PACU, similarly noted that more than three-quarters of complications involved patients categorised as ASA physical status I or II. ${ }^{(4)}$ The large number of critical incidents involving such 
Table IV. Critical incidents in the post-anaesthesia care unit.

\begin{tabular}{|c|c|}
\hline Category of incident & No. (\%) \\
\hline \multicolumn{2}{|l|}{ Cardiovascular $(n=293)$} \\
\hline Tachycardia & $113(38.6)$ \\
\hline Hypotension & $85(29.0)$ \\
\hline Arrhythmia & $31(10.6)$ \\
\hline Hypertension & $28(9.6)$ \\
\hline Myocardial ischaemia/chest pain & $19(6.5)$ \\
\hline Pulmonary oedema & $9(3.1)$ \\
\hline Bradycardia & $6(2.0)$ \\
\hline Cardiac arrest & $2(0.7)$ \\
\hline \multicolumn{2}{|l|}{ Respiratory $(n=155)$} \\
\hline Hypoxia & $103(66.5)$ \\
\hline Carbon dioxide retention & $41(26.5)$ \\
\hline Bronchospasm & $6(3.9)$ \\
\hline Pneumothorax & $4(2.6)$ \\
\hline Aspiration & $1(0.6)$ \\
\hline \multicolumn{2}{|l|}{ Airway-related $(n=34)$} \\
\hline Reintubation & $25(73.5)$ \\
\hline Obstruction & $7(20.6)$ \\
\hline Laryngospasm & $2(5.9)$ \\
\hline \multicolumn{2}{|l|}{ Neurological $(n=52)$} \\
\hline Reduced consciousness & $34(65.4)$ \\
\hline Agitation & $9(17.3)$ \\
\hline Seizures & $5(9.6)$ \\
\hline Neurological deficit & $3(5.8)$ \\
\hline $\begin{array}{l}\text { Spinal sensory level above T8, delayed recovery } \\
\text { from spinal anaesthesia }\end{array}$ & $1(1.9)$ \\
\hline \multicolumn{2}{|l|}{ Surgery-related $(n=47)$} \\
\hline Bleeding & $37(78.7)$ \\
\hline Other surgical complications & $10(21.3)$ \\
\hline \multicolumn{2}{|l|}{ Other $(n=120)$} \\
\hline Hypothermia & $37(30.8)$ \\
\hline Metabolic acidosis & $28(23.3)$ \\
\hline Allergy & $14(11.7)$ \\
\hline Hyperglycaemia/hypoglycaemia & $12(10.0)$ \\
\hline latrogenic patient injury & $9(7.5)$ \\
\hline Emesis & $8(6.7)$ \\
\hline Oliguria & $7(5.8)$ \\
\hline Drug error & $5(4.2)$ \\
\hline
\end{tabular}

patients in our study might also be partly due to the fact that the majority of our surgical patients were of ASA physical status I or II. Furthermore, in our institution, the general practice for patients requiring intensive postoperative care is to transfer them directly from the OT to the ICU, thereby reducing the number of patients with ASA physical status III and IV categories in the PACU.

The majority of critical incidents identified in our study were seen in patients who underwent general surgery. These patients had a higher incidence of PACU complications when compared to those from other disciplines $(p<0.001)$. This is in keeping with other studies, which have reported that patients undergoing major open abdominal procedures had a greater number of complications and longer mean length of stay in the PACU. ${ }^{(4,6,8)}$
Table V. Details of patients who suffered cardiac arrests in the post-anaesthesia care unit $(n=2)$.

\begin{tabular}{lll}
\hline Variable & Patient 1 & Patient 2 \\
\hline Age (yr) & 60 & 71 \\
ASA physical & III & III \\
status & & \\
Comorbidities & Diabetes mellitus, & Hypertension, \\
& pulmonary tuberculosis, & hyperlipidaemia, \\
& anaemia, gastro- & ischaemic heart \\
& oesophageal reflux disease & disease \\
& First and second toe ray & Exploratory \\
Procedure & amputation, excision of & laparotomy, total \\
& extensor tendons third, & proctocolectomy \\
& fourth and fifth toes & and end ileostomy \\
& Asystole & Ventricular \\
Initial rhythm & fibrillation \\
& Death & Death \\
\hline
\end{tabular}

ASA: American Society of Anesthesiologists

Table VI. Discharge disposition of patients with critical incidents in the PACU $(n=364)$.

\begin{tabular}{lc}
\hline Disposition & No. (\%) \\
\hline Ward & $51(14.0)$ \\
HD & \\
$\quad$ Planned & $83(22.8)$ \\
$\quad$ Unplanned & $120(33.0)$ \\
ICU & \\
Planned & $10(2.7)$ \\
Unplanned & $64(17.6)$ \\
Return to operation theatre & $34(9.3)$ \\
Death in PACU & $2(0.5)$ \\
\hline
\end{tabular}

HD: high dependency area; ICU: intensive care unit; PACU: post-anaesthesia care unit

Patients who underwent vascular surgery were also more likely to have a critical incident in the PACU $(p<0.001)$. Vascular surgery is associated with an increased perioperative cardiac risk. ${ }^{(9)}$ In addition, vascular surgeons at our institution perform a high volume of access work, and many of the patients undergoing these procedures have multiple and potentially complex comorbidities. Patients from the orthopaedics and obstetrics/ gynaecology disciplines were less likely to have a critical incident $(\mathrm{p}<0.001)$. This may also be explained by institution-specific factors. Patients who are scheduled for obstetrics/gynaecology procedures at our institution are generally young and well. Similarly, the orthopaedics department performs a large amount of elective procedures and less trauma-related surgery.

The most common critical incidents observed in the present study were cardiovascular-related $(41.8 \%)$ followed by respiratory $(22.1 \%)$, neurological $(7.4 \%)$, surgical $(6.7 \%)$ and airway-related (4.9\%). Specifically, tachycardia, hypotension, hypoxia and $\mathrm{CO}_{2}$ retention were the most common physiological derangements. Kluger and Bullock, who extracted 419 incidents from the Australian Incident Monitoring Study, also noted that the majority of their critical incidents were cardiorespiratory-related. ${ }^{(6)}$ The most frequently encountered problems in their study were 
associated with respiratory/airway issues (43\%), cardiovascular problems $(24 \%)$ and drug errors $(11 \%){ }^{(6)}$ Of note, Rose et al found that hypertension and tachycardia in PACU patients were associated with increased risk of unanticipated ICU admission and mortality. ${ }^{(10)}$

In our study, $50.5 \%$ of patients ( $\mathrm{n}=184$; equivalent to $0.4 \%$ of all major OT patients over the study period) required a transfer to a higher level of care (HD and ICU) than initially planned. Specifically, there were 64 (17.6\%) unplanned ICU admissions. Kluger and Bullock noted that $29 \%$ of all critical incidents that they reviewed resulted in unplanned admissions of patients to the HD or ICU. ${ }^{(6)}$ Another study involving 18,437 consecutive patients recorded a total of 186 unexpected ICU admissions. ${ }^{(4)}$ Critical incidents in the PACU influence postoperative resource utilisation in hospitals due to unexpected admissions of postoperative patients to higher levels of care.

Reintubations were required for 25 (6.9\%) of the 364 PACU patients, among whom 21 patients required unplanned ICU admission for ventilation. In their study, Kluger and Bullock reported that $17 \%$ of patients with a critical incident in the PACU required assistance with ventilation. ${ }^{(6)}$ Rose et al found that only $0.1 \%$ $(n=22)$ of patients among 21,457 consecutive general anaesthesia patients in the PACU required emergency reintubation. ${ }^{(7)}$ Similarly, Hines et al reported that 21 of 1,275 patients receiving upper airway support required reintubation, ${ }^{(4)}$ and Peskett et al found that $0.08 \%$ of 13,266 cases required reintubation. ${ }^{(11)}$ In comparison, the rate of emergency intubations in the PACU at our centre was $0.05 \%$ (25 in 49,532 procedures).

As a retrospective study of an administrative database, the present study had certain limitations. Not all data was uniformly available and no assumptions were made about missing data. The critical incidents were recorded in prose form, and therefore the investigators used clinical judgement while translating these reports into data. Although ambiguities were resolved through discussion between investigators, this may not have corrected for all inherent bias. In the context of a busy PACU, the administrative nature of the database may also mean that not all critical incidents were uniformly recorded during the study period. Therefore, the calculated incidence of critical events may be an underestimation of the actual number of incidents. We recommend that a prospective follow-up trial be undertaken, with data on critical incidents being collected by clinicians, to strengthen the findings of this study.
Despite the limitations, our study data highlights the role of a PACU in reducing patient morbidity in the immediate postoperative period. Without a PACU, many of these critical incidents would have occurred in the wards, where staff may not be in a position to manage such complications promptly. ${ }^{(6)}$ PACU staff must be highly skilled and receive ongoing training and education to be able to manage both a diverse range of complications and critically ill patients. ${ }^{(6)}$ In cases where critically ill patients are cared for in the PACU due to a shortage of beds elsewhere, the level of care provided must be on par with that provided in higher-level care units, such as the HD and ICU.

In conclusion, the current review documents the various critical incidents that were recorded over a two-year period in a major tertiary hospital and highlights the importance of a PACU in managing patients in the postoperative period. Critical incidents in the PACU can impact healthcare utilisation by prolonging patients' length of stay in the PACU and resulting in the need for higher levels of postoperative care than anticipated. Regular audits of critical incidents in the PACU are important to maintain the quality of immediate postoperative care, particularly in the context of an ageing population.

\section{REFERENCES}

1. Waddle JP, Evers AS, Piccirillo JF. Postanesthesia care unit length of stay: quantifying and assessing dependent factors. Anesth Analg 1998; 87:628-33.

2. Truong L, Moran JL, Blum P. Post anaesthesia care unit discharge: a clinical scoring system versus traditional time-based criteria. Anaesth Intensive Care $2004 ; 32: 33-42$.

3. Zelcer J, Wells DG. Anaesthetic-related recovery room complications. Anaesth Intensive Care 1987; 15:168-74.

4. Hines R, Barash PG, Watrous G, O'Connor T. Complications occurring in the postanesthesia care unit: a survey. Anesth Analg 1992; 74:503-9.

5. Bothner U, Georgieff M, Schwilk B. The impact of minor perioperative anesthesia-related incidents, events, and complications on postanesthesia care unit utilization. Anesth Analg 1999; 89:506-13.

6. Kluger MT, Bullock MF. Recovery room incidents: a review of 419 reports from the Anaesthetics Incident Monitoring Study (AIMS). Anaesthesia 2002; 57:1060-6.

7. Rose DK. [Recovery room problems or problems in the PACU]. Can J Anaesth 1996; 43(5 Pt 2):R116-28. English, French.

8. Seago JA, Weitz S, Walczak S. Factors influencing stay in the postanesthesia care unit: a prospective analysis. J Clin Anesth 1998; 10:579-87.

9. Fleisher LA, Beckman JA, Brown KA, et al. 2009 ACCF/AHA focused update on perioperative beta blockade incorporated into the ACC/AHA 2007 guidelines on perioperative cardiovascular evaluation and care for noncardiac surgery: a report of the American college of cardiology foundation/American heart association task force on practice guidelines. Circulation 2009; 120:e169-276.

10. Rose DK, Cohen MM, DeBoer DP. Cardiovascular events in the postanesthesia care unit: contribution of risk factors. Anesthesiology 1996; 84:772-81.

11. Peskett MJ. Clinical indicators and other complications in the recovery room or postanaesthetic care unit. Anaesthesia 1999; 54:1143-9. 